efficient segregation based on molecular typing of Pseudomonas aeruginosa would seem be the only appropriate management strategy for our cystic fibrosis patients.

\section{D.W. Reid*, A. Champion", R. Bradbury ${ }^{\#}$, S.M. Kirov}

Depts of *Respiratory Medicine and ${ }^{*}$ Pathology, University of Tasmania Medical School, Hobart, Tasmania, Australia.

\section{References}

1. Jones AM, Martin L, Bright-Thomas RJ, et al. Inflammatory markers in cystic fibrosis patients with transmissible Pseudomonas aeruginosa. Eur Respir J 2003; 22: 503506.

2. Armstrong DS, Nixon GM, Carzino R, et al. Detection of a widespread clone of Pseudomonas aeruginosa in a pediatric cystic fibrosis clinic. Am J Respir Crit Care Med 2002; 166: 983-987.

\section{From the authors:}

D.W. Reid and colleagues state that there is anecdotal evidence that the host inflammatory response at the time of acquisition may be what determines subsequent outcome, and propose that the results of our own study may be influenced by a survivor effect. However, unlike the clinical situation of a rapid deterioration and death sometimes seen among a proportion of cystic fibrosis (CF) patients who develop infection with transmissible strains of Burkholderia cenocepacia, we have not observed a similar rapid decline in the patients who have recently become infected by a transmissible Pseudomonas aeruginosa. D.W. Reid and colleagues also reflected on the experience of ARMSTRONG et al. [1]. However, there are likely to be differences between our own experience and that of ARMSTRONG et al. [1]. Firstly, ours is an adult CF centre, whilst the report of ARMSTRONG et al. [1] concerns paediatric $\mathrm{CF}$ patients. Secondly, the vast majority of our patients were infected with sporadic $P$. aeruginosa strains before acquiring a transmissible strain; it is likely that the $\mathrm{CF}$ patients in the report by ARMSTRONG et al. [1] were all previously $P$. aeruginosa naïve before developing infection with their clonal strain. Finally, it is possible that individual transmissible $P$. aeruginosa strains may differ in their pathogenicity.

It is clear that prospective studies of the clinical outcome for cystic fibrosis patients who harbour transmissible strains of Pseudomonas aeruginosa are needed before we can reach any firm conclusions of the clinical effects of Pseudomonas aeruginosa cross-infection in cystic fibrosis. We firmly agree with D.W. Reid and colleagues that until there is evidence to the contrary, cross-infection control measures should include segregation based upon the results of molecular fingerprint typing of isolates.

A.M. Jones, R.J. Bright-Thomas, M.E. Dodd, A.K. Webb Manchester Adult Cystic Fibrosis Centre, South Manchester University Hospitals NHS Trust, Manchester, UK.

\section{References}

1. Armstrong DS, Nixon GM, Carzino R, et al. Detection of a widespread clone of Pseudomonas aeruginosa in a pediatric cystic fibrosis clinic. Am J Respir Crit Care Med 2002; 166 : 983-987.

\title{
The use of computer-animation programs during spirometry in preschool children
}

\section{To the Editor:}

We read with interest the paper by GRACCHI et al. [1] describing the use of computer-animation programs as a device to improve reproducibility in acquiring forced expiratory manoeuvres in 4-8-yr-olds. The study was simple and well designed but the possibility both that the computeranimation programs were not used to their full potential and that the European Respiratory Society "adult" reproducibility criteria are too strict for preschool children must be raised $[2,3]$.

While we agree that the use of computer-animation incentive programs offers little advantage in the $6-8$-yr-old group, younger children cannot always understand, process and carry out multistep tasks. In such children, using a combination of the computer-animation programs will allow the production of a prolonged forceful expiration to be broken down into smaller steps. We find starting with the candles, to achieve maximal peak flow, and then progressing to the balloons or bowling alley games (prolonging the forced expiration) with individualised target modification provides maximum encouragement for each child according to their ability and lung function.
Individualised target modification is necessary as the animations are preset at a target of $120 \%$ predicted for both peak expiratory flow and forced vital capacity, a target that is only appropriate if based on suitable reference data for that age group. Any underestimation of predicted values (which, given the paucity of data from healthy $4-8$-yr-olds, is quite likely to occur) will mean that the child reaches the animation target before they have truly reached their maximum, thereby losing the incentive to try any harder as they will not be able to see any improvement in their performance. Likewise, on some occasions it may be appropriate to reduce the target $\%$ initially in order to encourage the younger children or those with poor coordination to aim for a target that is achievable, thereby encouraging them to try harder next time, and to prevent them giving up.

When comparing the proportion of children in the study by GRACCHI et al. [1] who were able to achieve certain reproducibility criteria under the different measurement conditions it is important to note the following: 1) none of the observed differences were significant; 2) most of the children produced forced expiratory parameters within $<0.1 \mathrm{~L}$, rather than within $5 \%$ of each other, the former equating to a 